\title{
Osteolysis and Cervical Cord Compression Secondary to Silicone Granuloma Formation around a Dorsal Spinal Cord Stimulator: A Case Report
}

\author{
John R. Dimar II ${ }^{1}$ David T. Endriga ${ }^{1} \quad$ Leah Y. Carreon ${ }^{1}$ \\ ${ }^{1}$ Norton Leatherman Spine Center, Louisville, Kentucky, \\ United States \\ J Neurol Surg Rep 2016;77:e67-e72. \\ Address for correspondence Leah Y. Carreon, MD, MSc, Norton \\ Leatherman Spine Center, 210 East Gray Street, Suite 900, Louisville, \\ Kentucky, 40202, United States \\ (e-mail: leah.carreon@nortonhealthcare.org).
}

\begin{abstract}
Spinal cord stimulators (SCSs) have long been in use as a modality for the management of numerous pain pathologies. Along with commonly anticipated morbidities such as displacement, failure (due to fracture or breakage), or infection, there have also been rare but well-documented complications of fibrous scarring, resulting in spinal cord compression. This is the first known case that demonstrates osteolysis and bony destruction of the vertebrae adjacent to the SCS along with the foreign-body granulomatous reaction. A 61-year-old man who underwent prior posterior cervical implantation with an SCS followed by multiple revisions presented with progressive paresthesias, numbness, and weakness of his upper extremities 10 years later. The SCS was removed

Keywords

- spinal cord stimulator complications

- silicone granuloma

- osteolysis secondary to silicone implants followed by decompression, and instrumented fusion of the cervical spine. Histopathologic analysis reveals foreign-body reaction to the SCS and its silicone debris. Tissue cultures were negative for bacterial, fungal, or mycobacterial infection. No malignancy was seen. The current case illustrates the inherent possibility of foreign-body granulomatous reactions with SCS and its silicone particulate matter, made unique in this instance by the associated bony destruction of the adjacent vertebrae.
\end{abstract}

\section{Introduction}

Since it was first used in 1967, spinal cord stimulation (SCS) has become a commonly used procedure for the management of intractable pain arising from such conditions as failed back surgery syndrome, complex regional pain syndrome, refractory angina, phantom limb pain, postthoracotomy pain syndrome, and reflex sympathetic dystrophy. As with any implanted device, complications would be anticipated. Some of the more common complications include electrode migration, hardware malfunction, cerebrospinal fluid (CSF) leakage, infection, and hematoma. Nerve root/spinal cord injury, allergic reactions, and skin erosions have also been reported but are less frequently encountered. ${ }^{1}$

received

October 13, 2015

accepted

December 30, 2015
DOI http://dx.doi.org/

10.1055/s-0036-1579631. ISSN 2193-6358.
One of the rarest complications involves the formation of a mass surrounding the SCS electrode, resulting in spinal cord compression. A search of the published literature showed that only seven documented cases have been reported. ${ }^{2-7}$ In each case the SCS was implanted for various intractable pain conditions that initially resolved but were later characterized by decreasing pain control (tolerance) and progressive neurological deficits (weakness, spastic quadriparesis among others). Neurologic deficits improved after removal of the SCS and decompression of the spinal cord and removal of the accumulated scar tissue.

This case report deals with one such complication, wherein a SCS peri-implant mass resulted in cervical spinal cord compression. It is distinguished, however, by the presence of lytic,

\footnotetext{
(c) 2016 Georg Thieme Verlag KG Stuttgart · New York
}

License terms

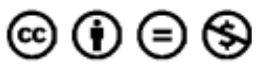


expansile bony lesions in the structures adjacent to the implant, and the resulting layers of scar tissue around the electrode causing spinal cord compression. None of the previous cases described in literature have made mention of encountering such a complication of osteolysis of the adjacent vertebral structures adjacent to the tissue surrounding the SCS causing spinal cord compression. Similar findings, however, have been encountered predominantly in silicone containing hand implants, but none so far regarding the spine.

\section{Case Report}

The patient is a 61-year-old man who presented with a chief complaint of gradual-onset neck pain, 6/10 in intensity, aching in character, which had progressively been getting worse over the course of 6 months. He also complained of worsening paresthesias, numbness, and weakness of his upper extremities along with decreased grip strength of both hands. No radicular pain was noted. His pertinent past medical history reveals that he had previously been diagnosed with reflex sympathetic dystrophy for which he was treated with implantation of posterior cervical dorsal spinal cord stimulators around 10 years prior. This was implanted twice followed by four revisions, and later followed by implantation of a Codman pain pump in 2009, which was then revised in 2012 .
On physical examination, there were no significant findings apart from weakness of grip strength on the left hand, diffuse hand numbness, and positive Hoffman reflex on the left. There was midline and paraspinous tenderness of the posterior cervical spine on examination. He had decreased cervical kyphosis and his head was sagittally displaced forward.

\section{Imaging and Laboratory Findings}

Computed tomographic (CT) scan of the cervical spine (-Fig. 1) showed the paddles of the spinal cord stimulator in place with evidence of the prior wide laminectomies done at C2-C3 and a partial laminectomy of the superior portion of the C4 lamina. Spinal cord flattening and volume loss was also seen extending from $\mathrm{C} 2-\mathrm{C} 3$ to $\mathrm{C} 3-\mathrm{C} 4$ with associated central and foraminal stenosis at these levels. There were also expansile, lytic bony lesions of the vertebral bodies and the lateral masses of $\mathrm{C} 4$ and $\mathrm{C} 5$ that were initially considered to be possibly metastatic cancer or a primary bone tumor. The differential diagnosis included infection (bacterial or tuberculosis), hemangioma, or Paget disease. Given the presence of the dorsal column stimulator electrode, a histolytic foreignbody reaction could also not be ruled out. No fractures, anterolisthesis, or paravertebral masses were noted. Preoperative white blood count (WBC) count was within normal range at 9.48 , with the differential counts within normal
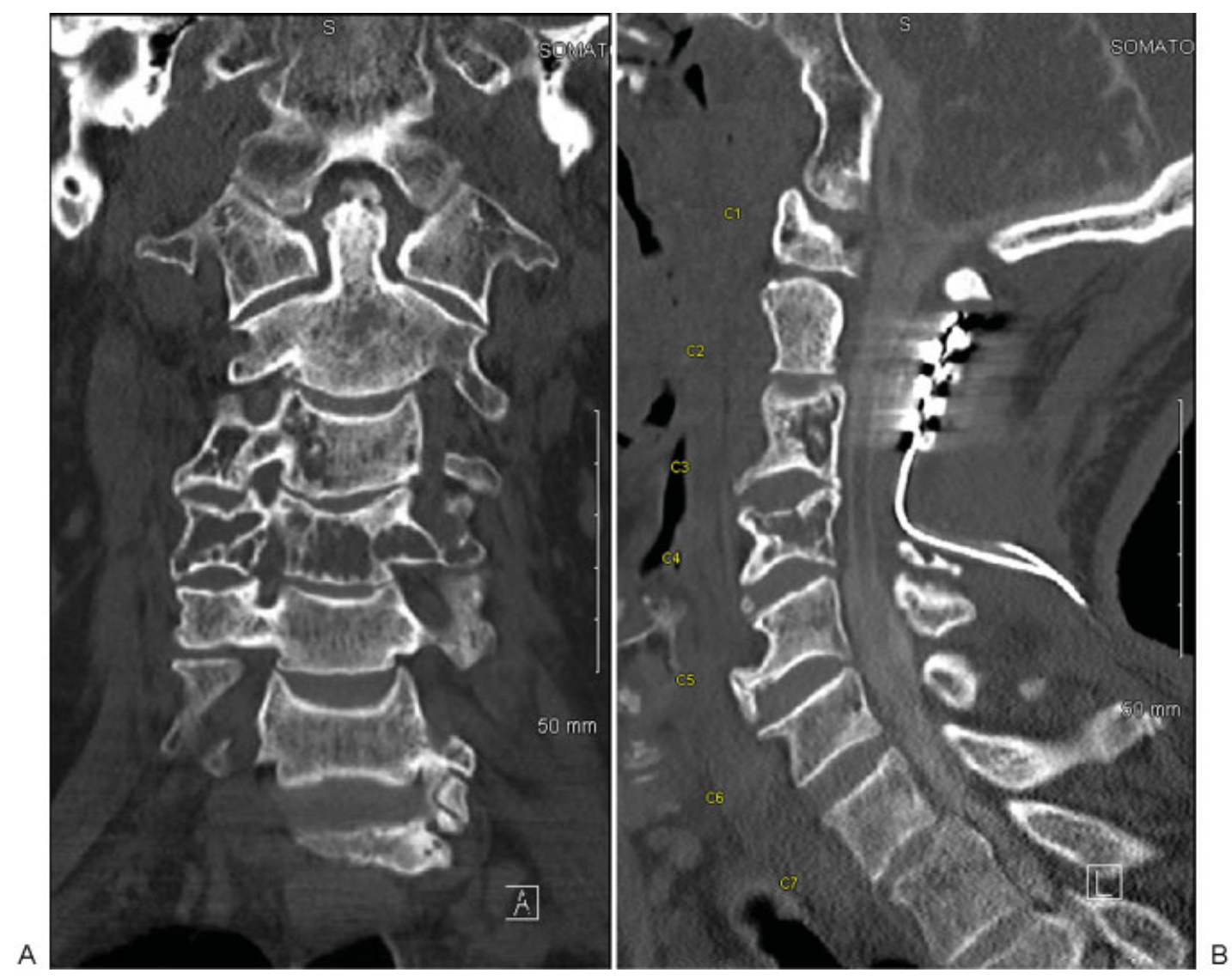

Fig. 1 Computed tomography of the cervical spine. (A) Frontal reconstruction showing a lytic lesion the vertebral body and lateral masses of C3 and C4. (B) Sagittal reconstruction showing flattening of the spinal cord. 
parameters. Erythrocyte sedimentation rate was slightly elevated at $17 \mathrm{~mm} / \mathrm{h}($ normal $=0-15 \mathrm{~mm} / \mathrm{h})$ and C-reactive protein was elevated at $24.6 \mathrm{mg} / \mathrm{L}$ (normal $\leq 10 \mathrm{mg} / \mathrm{L}$ ).

A two-staged management plan was determined to be best considering the unknown nature of the lesions. The first stage was to remove the implant, decompress the spinal canal, debride the bone, and send samples for histiologic examination and a stat Gram stain. Additionally, the plan was to procure cultures, including aerobic, anaerobic, fungal, tuberculosis, propriobacter both swab and tissue, followed by a posterior spinal fusion with instrumentation using iliac crest bone graft procured prior to making the neck incision. The second staged procedure planned was an instrumented anterior vertebrectomy, decompression, and plating.

The dorsal spinal cord stimulator was removed through the prior posterior cervical incision. The implant was meticulously dissected off the dura, taking care to avoid any tears. The electrode was densely encapsulated in very firm layers of fibrous tissue. Following removal of the electrodes, the spinal cord was decompressed by carefully dissecting multiple fine layers of scar tissue that had formed over the laminectomy sites. The remaining lamina and lateral masses were exposed, and it was noted that the lateral masses of C3 and C4 were eroded with a thinned out cortex and perforations through it. There was soft pliable tissue, almost purulent in nature curetted out of the defects. The lateral mass of both vertebras on the left was extensively destroyed by the process and C4 vertebra was essentially hollow following debridement. Numerous swab and tissue specimens were collected and sent for a stat Gram stain and histopathologic, bacterial, fungal, and mycobacterial analysis. Copious irrigation of the wound was done following the debridement. The stat Gram stain was negative and the frozen section did not show malignancy, and only granulomas were noted. Because an acute pyogenic infection did not appear and the spine was destabilized by the surgery, it was elected to instrument it concurrently with the decompression using lateral mass screws from $\mathrm{C} 2$ to $\mathrm{C}$, skipping screw placement in the destroyed left C4 lateral mass, and then meticulously autografted with iliac crest bone graft that was harvested prior to the neck incision. Vancomycin powder was placed in the wound that was followed by insertion of deep and superficial drains and closure of the incision.

In the interim period, the fungal, acid-fast bacilli (AFB), and bacterial cultures were negative and final histologic analysis showed no malignancy. Tissue analysis of the fibrocartilage, bone, and muscle specimens showed foamy and hemosiderin-laden macrophages, cholesterol clefts, and foreign-bodytype giant cell reaction and non-necrotizing granulomas. The pathologist also noted numerous particles of silicone within the granulomas and reported that the histology most likely represented a foreign-body histolytic reaction.

The anterior procedure was done 5 days later and consisted of a standard C4 vertebrectomy where the disc above and below C4, followed by excision of the vertebra using rongeurs and curettes, sending all of the material for histopathologic analysis, bacterial, fungal, and mycobacterial cultures. Intraoperatively, the C4 vertebra was significantly involved with the destructive process while there was only partial destruction of $\mathrm{C} 3$ whose endplates were still intact. A titanium metallic cage was then measured to fit into the gap left by the $C 4$ vertebrectomy and was packed with the allograft. It was then tapped into place flush with the anterior vertebral body margins. A 45-mm Venture plate (Medtronic, Memphis, TN) was then placed over the cage, spanning from $\mathrm{C} 3$ to $\mathrm{C} 5$, held in place with 50 $\times 4.0-\mathrm{mm}$ screws that were noted to have good purchase. The wound was then washed and drains were inserted followed by closure in layers (-Fig. 2).
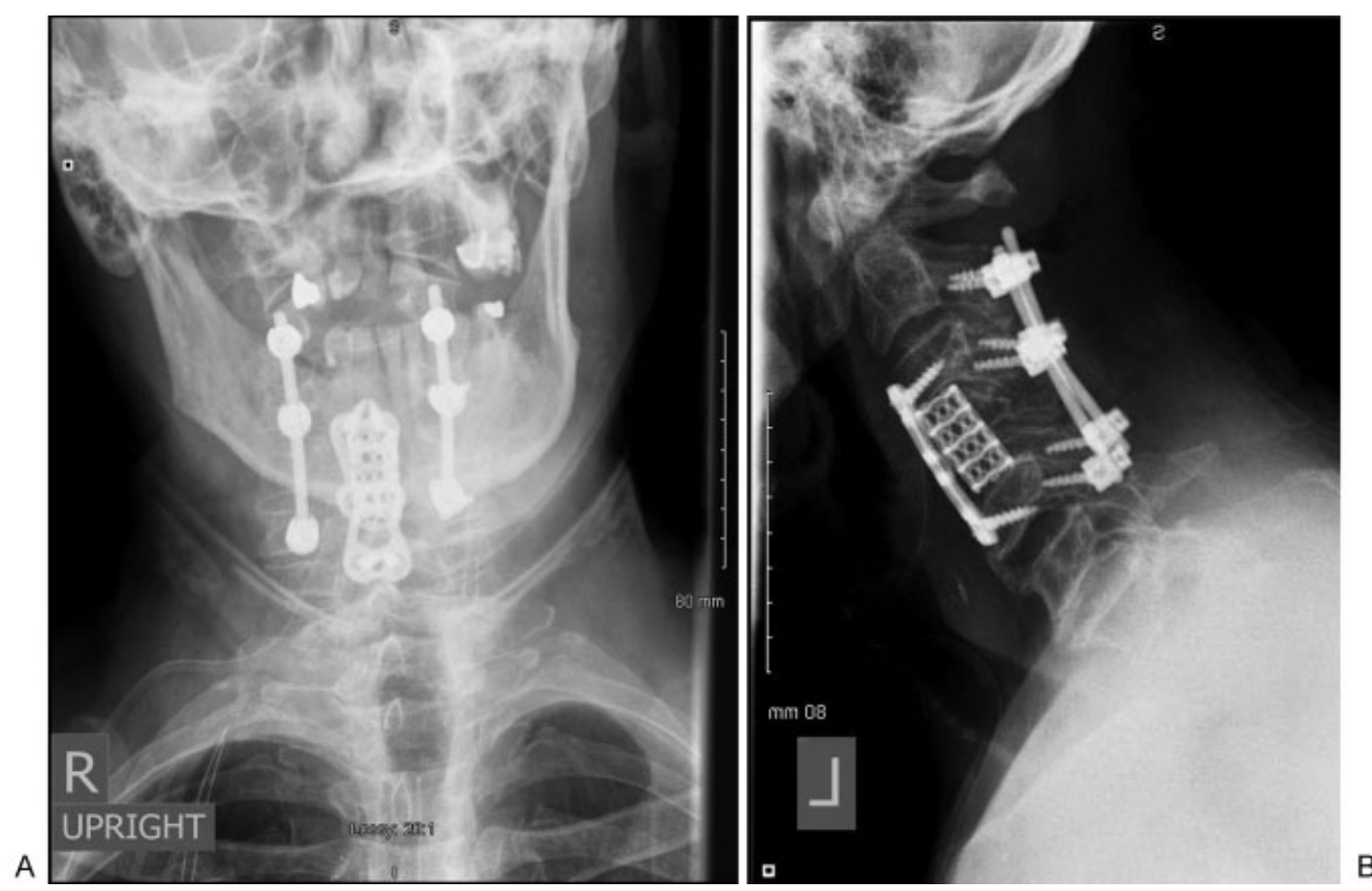

Fig. 2 Postoperative cervical (A) anteroposterior and (B) lateral radiographs showing lateral mass instrumentation and interbody cage. 


\section{Histopathology Findings}

The biopsy specimens gathered in the first procedure on the posterior cervical spine revealed foamy and hemosiderinladen macrophages, cholesterol clefts, and foreign-body-type giant cell reaction ( $\mathbf{- F i g . 3 A}$ ). These were also negative for malignancy, and cultures for fungi, AFB, and aerobic and anaerobic bacteria were likewise negative. The intraoperative frozen sections likewise revealed the presence of giant cells without any malignancies identified.

Specimens collected from the second procedure 5 days later consisting of bone fragments and articular cartilage revealed necrotizing ( - Fig. 3B) and non-necrotizing (-Fig. 3C) granulomatous inflammation. Foreign-body particles were also visualized under polarized light ( - Fig. 3D). Silicone particles were located inside the granulomas and were found both posteriorly in the lateral mass and in the anterior vertebra, probably by contiguous spread from the posterior lateral mass area where the lamina/lateral mass was lysed. As with the previous histopathology and tissue culture reports, this specimen was also negative for malignancy with no growth of fungi, AFB, or bacteria.

\section{Follow-up}

One year after surgery, the incisions are well-healed, and the patient has limited cervical range of motion and decreased grip strength on the left, 5/5 motor strength for both writs flexion and extension. The patient no longer complains of numbness or paresthesias over both upper extremities. Radiographs show no subsidence or loosening of the implants (-Fig. 4).

\section{Discussion}

The previously mentioned histopathologic results are consistent with the findings of a foreign-body reaction, more specifically with that of a silicone granulomatous reaction. This, along with the absence of infection, is consistent with the finding of macrophages and foreign-body giant cells described in other cases of silicone foreign-body reactions. ${ }^{8-10}$ Though the findings of a silicone granulomatous reaction may have been established, the exact mechanism of granuloma formation is as yet unclear. We had noted on the preoperative $\mathrm{CT}$ scan that the lytic areas in the lateral mass communicated with the lytic areas in the anterior body of the vertebra. We believe that this was the potential pathway that the silicone particles gradually spread through as the granulomatous reaction occurred, resulting in the lytic areas in the vertebra anteriorly. We hypothesize that this is the most logical explanation of why we were able to recover granulomas in the anterior body that demonstrated embedded silicone particles. Lopiccolo et al have mentioned that it
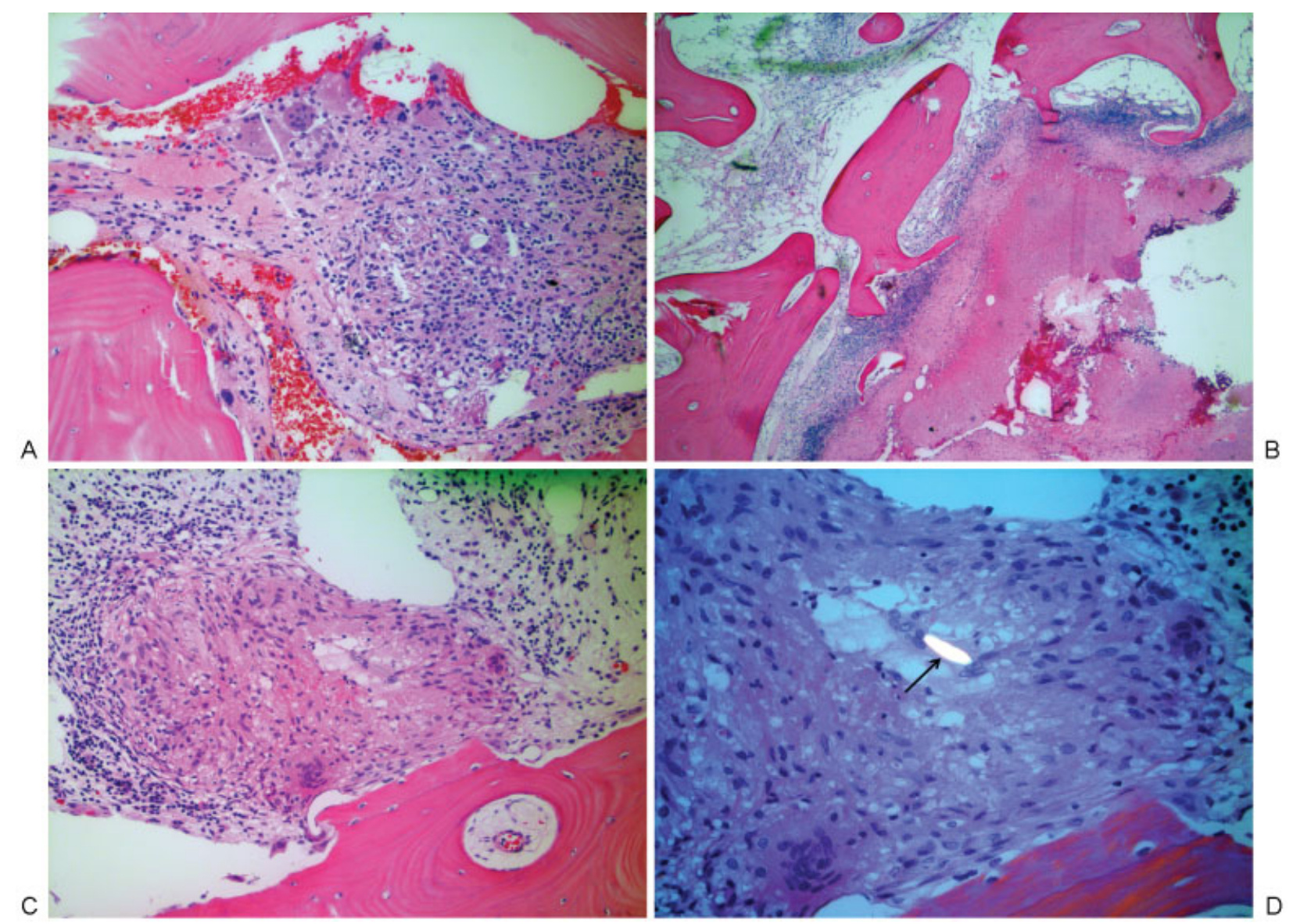

Fig. 3 Histopathologic specimens showing (A) foreign-body giant cells, (B) necrotizing granulomatous inflammation, (C) non-necrotizing granulomatous inflammation with foreign-body-type giant cells, (D) foreign body (silicone particle) visualized under polarized light. 
A

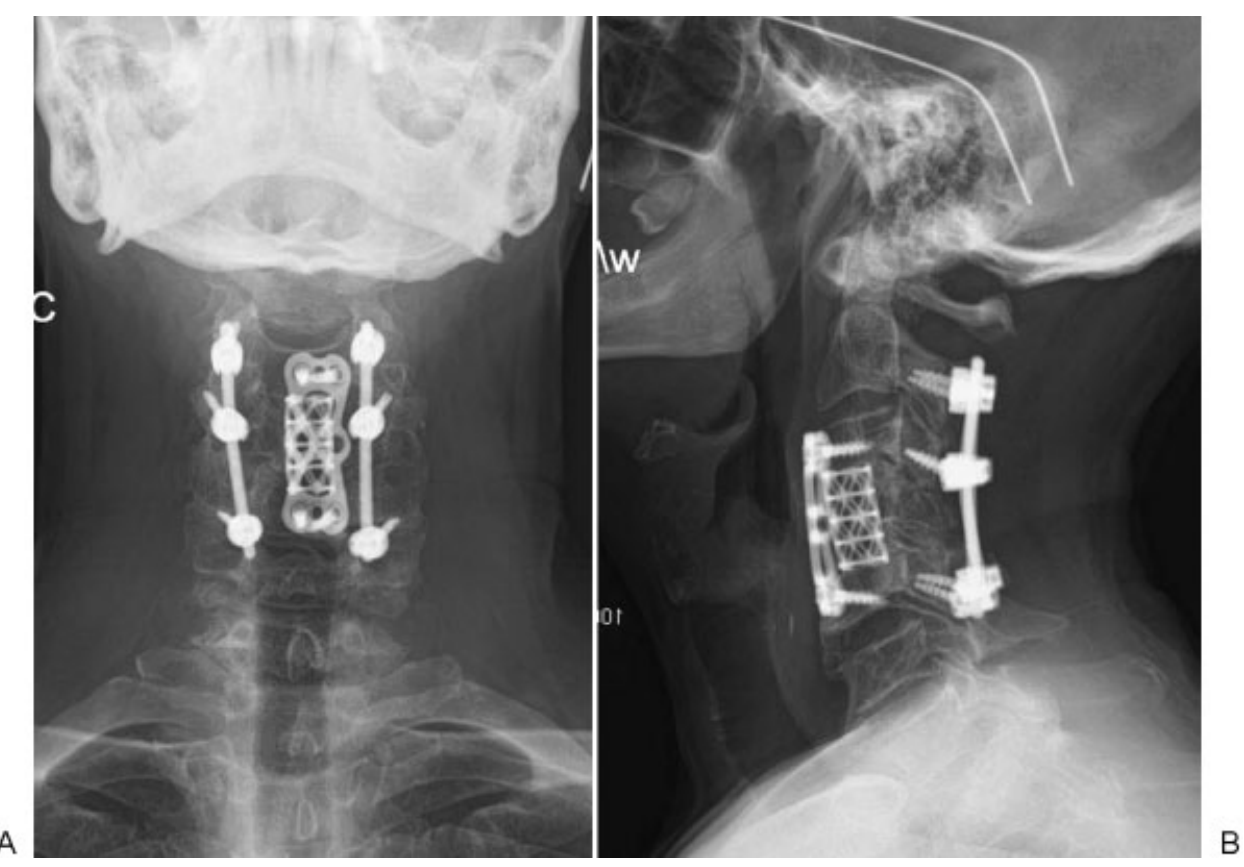

Fig. 4 One-year postoperative cervical (A) anteroposterior and (B) lateral radiographs showing fusion across the lateral mass posteriorly and the vertebral bodies anteriorly.

may originate with a trigger (infection, trauma, adulterants) that sets of a T-cell reaction that releases cytokines, thus leading to granuloma formation. ${ }^{10} \mathrm{He}$ also makes mention of the silicone serving as a possible nidus for bacteria from different sites, where they may colonize the silicone by means of biofilm. The resulting coordinated gene expression my confer resistance to antibiotics and immune defenses. This resistance may also increase due to the decreased local perfusion at the silicone implant site. ${ }^{10}$

The presence of a perielectrode mass composed of thickened scar tissue in our patient may be confirmed by the possible presence of a tolerance phenomenon during the course of use of the spinal cord stimulator. It was elicited from his past medical history that he had previously undergone multiple revisions of his SCS, followed by the implantation of a Codman pain pump that would point to decreased pain control. The phenomenon of tolerance is one that has been previously documented in reports of patients who have had thickened fibrous scar tissue develop around their SCS, causing cord compression symptoms. ${ }^{6}$

What distinguishes this case, however, from previously reported cases is the lytic destruction of the bony structures adjacent to the sites of scar formation, more specifically, the vertebral bodies and lateral masses of $\mathrm{C} 4$. There have been previously reported cases of bony lytic destruction in the presence of silicone implants, but almost all of these have been associated with wrist arthroplasty implants. ${ }^{11-13}$ Despite prior cases of granulomatous scar formation with resulting spinal cord compression around SCS implants being reported, a thorough literature search has not yet yielded another report of osteolysis in the presence of an SCS foreignbody reaction, making this case unique in the literature.

The possible causes of this bone destruction may be related to cystic bone changes that can be seen in wrist arthroplasty with silicone implants, which depend mainly on silicone wear with reactive granulomatosis and is linked to implant load and deformation. ${ }^{13}$ This may be due to a reaction that occurs when there is fragmentation of the silicone in the implant, leading to subsequent immune-mediated bony destruction as a response to the silicone debris. ${ }^{8}$ This is confirmed by the findings on histopathology report that demonstrated the presence of foreign-body giant cells and granulomatous inflammation in the bony tissue specimens.

The management of our patient also distinguishes it from previously reported cases of perielectrode scar formation causing spinal cord compression. While most of them were managed with removal of the implant and decompression of the cord by removal of the scar tissue, the bony destruction of the vertebral bodies and lateral masses made it imperative that the cervical spine be stabilized with instrumented fusion both anteriorly and posteriorly. This was done in a staged manner following the findings with the posterior procedure that there were no signs of a malignant process or pyogenic infection. This allowed for the immediate stabilization of the neck posteriorly following removal of the implant and decompression. Following the findings from the final histologic analysis and cultures that demonstrated that no active infection was present, we proceeded with the anterior vertebrectomy to remove the vertebral body lesions, which showed identical findings that were seen posteriorly, followed by anterior stabilization.

This case highlights a rare cause of a granulomatous reaction to silicone in the setting of bone-silicone interphase. While current literature shows most cases are from dermatologic 8,14 and aesthetic ${ }^{9,10,14-16}$ procedures due to significantly larger numbers of use, it is still possible to encounter these cases wherever silicone interacts with human tissue, despite the rarity of these cases. In 
e72 Osteolysis and Cervical Cord Compression Secondary to Silicone Granuloma Formation Dimar II et al.

conclusion, it is important to include a possible silicone histolytic granulomatous reaction in the differential diagnosis when a silicone implant is adjacent to the spines osseous structures and lysis of the bone is observed on radiographs.

\section{References}

1 Bendersky D, Yampolsky C. Is spinal cord stimulation safe? A review of its complications. World Neurosurg 2014;82(6):1359-1368

2 Cicuendez M, Munarriz PM, Castaño-Leon AM, Paredes I. Dorsal myelopathy secondary to epidural fibrous scar tissue around a spinal cord stimulation electrode. J Neurosurg Spine 2012;17(6):598-601

3 Dam-Hieu P, Magro E, Seizeur R, Simon A, Quinio B. Cervical cord compression due to delayed scarring around epidural electrodes used in spinal cord stimulation. J Neurosurg Spine 2010;12(4):409-412

4 Lennarson PJ, Guillen FT. Spinal cord compression from a foreign body reaction to spinal cord stimulation: a previously unreported complication. Spine 2010;35(25):E1516-E1519

5 Reynolds AF, Shetter AG. Scarring around cervical epidural stimulating electrode. Neurosurgery 1983;13(1):63-65

6 Scranton RA, Skaribas IM, Simpson RK Jr. Spinal stimulator perielectrode masses: case report. J Neurosurg Spine 2015;22(1):70-74

7 Wada E, Kawai H. Late onset cervical myelopathy secondary to fibrous scar tissue formation around the spinal cord stimulation electrode. Spinal Cord 2010;48(8):646-648
8 Altmeyer MD, Anderson LL, Wang AR. Silicone migration and granuloma formation. J Cosmet Dermatol 2009;8(2):92-97

9 Bassetto F, Scarpa C, Caccialanza E, Montesco MC, Magnani P. Histological features of periprosthetic mammary capsules: silicone vs. polyurethane. Aesthetic Plast Surg 2010;34(4):481-485

10 Lopiccolo MC, Workman BJ, Chaffins ML, Kerr HA. Silicone granulomas after soft-tissue augmentation of the buttocks: a case report and review of management. Dermatol Surg 2011;37(5):720-725

11 Joyce TJ. Re: Grading of radiographic osteolytic changes after Silastic metacarpophalangeal arthroplasty and a prospective trial of osteolysis following use of Swanson and Sutter prostheses. J Hand Surg Eur Vol 2007;32(2):236-237, author reply 237

12 Hirakawa K, Bauer TW, Culver JE, Wilde AH. Isolation and quantitation of debris particles around failed silicone orthopedic implants. J Hand Surg Am 1996;21(5):819-827

13 Kistler U, Weiss AP, Simmen BR, Herren DB. Long-term results of silicone wrist arthroplasty in patients with rheumatoid arthritis. J Hand Surg Am 2005;30(6):1282-1287

14 Lemperle G, Gauthier-Hazan N, Wolters M, Eisemann-Klein M, Zimmermann U, Duffy DM. Foreign body granulomas after all injectable dermal fillers: part 1. Possible causes. Plast Reconstr Surg 2009;123(6):1842-1863

15 Anastassov GE, Schulhof S, Lumerman H. Complications after facial contour augmentation with injectable silicone. Diagnosis and treatment. Report of a severe case. Int J Oral Maxillofac Surg 2008;37(10):955-960

16 Dragu A, Theegarten $\mathrm{D}$, Bach $\mathrm{AD}$, et al. Intrapulmonary and cutaneous siliconomas after silent silicone breast implant failure. Breast J 2009;15(5):496-499 